\title{
DEFINIÇÃO DE METADADOS PARA RECURSOS INFORMACIONAIS: APRESENTAÇÃO DA METODOLOGIA BEAM
}

\section{DEFINICIÓN DE LOS METADATOS PARA RECURSOS DE INFORMACIONALES: PRESENTACIÓN DE LA METODOLOGÍA BEAM}

\begin{abstract}
Plácida Leopoldina Ventura Amorim da Costa Santos placida@marilia.unesp.br

Doutora em Semiótica e Linguística Geral pela Universidade de São Paulo (USP). Docente do Programa de Pós-Graduação em Ciência da Informação da Universidade Estadual Paulista (UNESP/Marília).

Ana Carolina Simionato - anacarolina.simionato@gmail.com Doutoranda em Ciência da Informação pela Universidade Estadual Paulista (UNESP/Marília). Docente do Departamento de Ciência da Informação da Universidade Estadual de Londrina (UEL).

Felipe Augusto Arakaki - fe.arakaki@gmail.com Graduando em Biblioteconomia pela Universidade Estadual Paulista
\end{abstract} (UNESP/Marília).

\section{RESUMO}

Introdução: No ambiente digital, os metadados influenciam tanto no acesso aos dados quanto na recuperação da informação e são utilizados como elementos de busca para facilitar o acesso e a recuperação de recursos na Web.

Objetivo: Nessa perspectiva, o objetivo é apresentar a metodologia BEAM, desenvolvida na Biblioteca de Estudos e Aplicação de Metadados, do Grupo de Pesquisa - Novas Tecnologias em Informação, da UNESP e utilizada para definir os metadados para a descrição de recursos informacionais.

Metodologia: Nesse sentido, a metodologia utilizada para a construção da pesquisa é 
Plácida Leopoldina Ventura Amorim da Costa Santos; Ana Carolina Simionato; Felipe Augusto Arakaki

Definição de metadados para recursos informacionais: apresentação da metodologia BEAM

exploratória e bibliográfica e foi desenvolvida tendo como base teórica o método de Chuttur (2011) e o ciclo de vida dos dados do DataONE (2012) e ainda o ciclo PDCA e a ferramenta $5 \mathrm{~W} 1 \mathrm{H}$.

Resultados: As sete etapas da metodologia são apresentadas e também as orientações necessárias para sua aplicação.

Conclusões: Conclui-se apontando que a metodologia BEAM pode ser adotada por bibliotecas, no processo de construção de catálogos voltados para o atendimento das necessidades dos usuários.

Palavras-chave: Metadados. Catalogação automatizada. Modelagem de catálogos. Definição de metadados. Informação e tecnologia.

\section{INTRODUÇÃO}

Com a expansão do uso das tecnologias de informação e comunicação e da globalização, a geração e o uso de informações crescem exponencialmente. Nesse contexto, faz-se necessário um tratamento adequado para as informações registradas que facilite o seu acesso, recuperação, uso, preservação e reuso, evitando perdas no fluxo informacional.

Grande parte da produção informacional está armazenada no ambiente da Web, entretanto, a dificuldade no processo de acesso, de busca e de recuperação de informações, se configuram em uma das preocupações da área da Ciência da Informação. A partir destes pressupostos a Ciência da Informação é definida por Borko (1968, p. 3, tradução nossa) como,

[...] uma ciência interdisciplinar que investiga as propriedades e o comportamento da informação, as forças que governam os fluxos e o uso da informação, e as técnicas tanto manual quanto mecânica de Processos de Informação para otimizar o armazenamento, a recuperação, e a disseminação da informação.

Assim, cabe aos profissionais dessa área, os bibliotecários, por exemplo, desenvolver metodologias e técnicas que possam tratar a informação apropriadamente. As metodologias e os métodos de tratamento da informação envolvem tanto o seu tratamento temático quanto o seu tratamento descritivo, ou seja, seu conteúdo e forma.

Os métodos, nos sistemas automatizados de indexação e de recuperação de recursos, estão cada vez mais sofisticados. Entretanto, a relevância e a precisão das 
Plácida Leopoldina Ventura Amorim da Costa Santos; Ana Carolina Simionato; Felipe Augusto Arakaki

Definição de metadados para recursos informacionais: apresentação da metodologia BEAM

respostas obtidas ainda não são satisfatórias, pois apenas dados não são suficientemente capazes para identificar um recurso informacional na Internet (BAPTISTA; MACHADO, 2001).

Glushko (2013) diz que:

O recurso possui um sentido comum de 'qualquer coisa de valor que pode apoiar a atividade orientada a um objetivo.' Esta definição significa que um recurso pode ser uma coisa física, ou uma coisa não-física, informações sobre coisas físicas, ou informações sobre coisas não-físicas, ou qualquer coisa que você quer organizar. Em outras palavras, o que direciona este amplo escopo são entidade, objeto, item, e instancia. O termo documento é frequentemente utilizado para um recurso informacional, seja ele em formato digital ou analógico (GLUSHKO, 2013, p. 8, grifo do autor, tradução nossa).

O termo recurso informacional refere-se a informação objetivada no contexto de um campo do conhecimento podendo ser apresentado em uma estrutura analógica e/ou digital, com valor informacional que caracteriza a sua concepção intelectual expressa na corporificação de manifestações estruturadas na forma de itens. O recurso informacional também é nominado de: 'item informacional', 'informação registrada', 'coisa física, ou uma coisa não-física' e 'artefato'.

Os recursos informacionais possuem características que requisitam um tratamento descritivo específico para otimizar sua busca, acesso e recuperação. Para tanto, os elementos descritivos representam as características de um recurso, que são denominados metadados e segundo Alves (2010, p. 47), podem ser descritos como,

[...] atributos que representam uma entidade (objeto do mundo real) em um sistema de informação. Em outras palavras, são elementos descritivos ou atributos referenciais codificados que representam características próprias ou atribuídas às entidades; são ainda dados que descrevem outros dados em um sistema de informação, com o intuito de identificar de forma única uma entidade (recurso informacional) para posterior recuperação.

No ambiente digital, os metadados influenciam tanto no acesso quanto na recuperação da informação e são utilizados como elementos de busca para facilitar o acesso aos dados descritivos e a localização de recursos na Web, e para que tenham aceitação internacional e auxiliem na interoperabilidade eles devem possuir 
Plácida Leopoldina Ventura Amorim da Costa Santos; Ana Carolina Simionato; Felipe Augusto Arakaki

Definição de metadados para recursos informacionais: apresentação da metodologia BEAM

uma padronização.

A padronização gera uma estrutura denominada como padrão de metadados, que segundo Alves (2010, p. 47-48) são:

[...] estruturas de descrição constituídas por um conjunto predeterminado de metadados (atributos codificados ou identificadores de uma entidade) metodologicamente construídos e padronizados. O objetivo do padrão de metadados é descrever uma entidade gerando uma representação unívoca e padronizada que possa ser utilizada para recuperação da mesma.

Os padrões de metadados garantem a possibilidade de troca de informações entre instituições, o que é de suma importância para o processo de representação informacional, pois sua utilização padronizada reduz o (re)trabalho na descrição de recursos informacionais (FUSCO, 2010).

Logo, a eficiência e a eficácia dos serviços de informação também são influenciadas pela utilização adequada de metadados, pois segundo Baptista e Machado (2001, p. 1),

De modo a melhorar a eficácia e a eficiência dos serviços de informação, torna-se necessária a utilização de metadados semânticos. No entanto, com a utilização de diferentes metadados e estruturas de metadados pelos diversos tipos de serviços e software, o problema mantém-se. Conforme acontece em outras áreas, a padronização é um elemento chave para a utilização eficaz dos metadados.

No momento da escolha do padrão de metadados, os catalogadores não devem considerar apenas as necessidades da instituição, mas principalmente, as necessidades informacionais dos usuários. E é nesse momento que verifica se esse padrão de metadados, em eleição, contempla as necessidades exigidas.

O propósito desse artigo é explicitar a importância da modelagem dos padrões de metadados na configuração dos sistemas automatizados, bem como apresentar uma metodologia construída sobre a definição de metadados para diferentes tipos de recurso, descrevendo a metodologia desenvolvida na Biblioteca de Estudos e Aplicação de Metadados (BEAM) ${ }^{1}$.

\footnotetext{
${ }^{1}$ Biblioteca de Estudos e Aplicação de Metadados (BEAM), um projeto do Grupo de Pesquisa - Novas Tecnologias em Informação (GP-NTI/Unesp), sob a coordenação da prof Dr$^{\text {a }}$ Plácida L. V. Amorim 
Plácida Leopoldina Ventura Amorim da Costa Santos; Ana Carolina Simionato; Felipe Augusto Arakaki

Definição de metadados para recursos informacionais: apresentação da metodologia BEAM

A metodologia BEAM favorece a criação e o planejamento da modelagem de catálogos e de sistemas informacionais subsidiando a construção de novos padrões de metadados, caso haja necessidades específicas em um determinado domínio do conhecimento ou necessidades específicas de um grupo específico de usuários.

No caso de criação de um novo padrão de metadados evidencia-se a necessidade de garantia da interoperabilidade entre os sistemas para a troca eficiente de informações. Nesse contexto, valerá colocar em prática o método equivalência dos dados, conhecido como Crosswalking (ST. PIERRE; LAPLANT, 1998) para manter o mínimo de correspondência entre metadados em diferentes padrões. Considera-se também, neste caso, as orientações de construção de Perfis de Aplicação disponíveis no padrão de metadados Dublin Core (COYLE; BAKER, 2009; NILSSON; BAKER; JOHNSTON, 2008) como exemplos de extensões do padrão Dublin Core desenvolvidas para domínios específicos.

\section{METADADOS}

Na década de 1960, Jack E. Myers, presidente e fundador da The Metadata Corporation cunhou o termo metadata (metadados) para descrever conjunto de dados que podem ser utilizados na organização, na representação e na localização de recursos e podem ser trabalhados de diferentes formas nas gestão de recursos informacionais e se constituem em unidades menores do que os dados que representam (SMIRAGLIA, 2005).

As bibliotecas vêm compartilhando metadados em sistemas informatizados, por meio do uso de esquemas e de padrões de descrição, tais como, os códigos de catalogação e os formatos de intercâmbio de dados. O termo metadados, entretanto, aparece com maior frequência na Biblioteconomia, na década de 80 (GILLILANDSWETLAND, 1999).

Os metadados explicitam os diferentes aspectos do recurso que descreve: sua estrutura, conteúdo, qualidade, contexto, origem, propriedade e condição. E auxiliam na organização, favorecem a interatividade, validam as identificações e

da Costa Santos com a participação de bolsistas FAPESP, CNPq, PROEX-Unesp e voluntários da graduação em Biblioteconomia e do Programa de Pós-Graduação em Ciência da Informação da UNESP, Campus de Marília (SANTOS; ALVES, 2014).

Inf. Inf., Londrina, v. 19, n. 1, p. $146-163$, jan./abr. 2014. http:www.uel.br/revistas/informacao/ 
Plácida Leopoldina Ventura Amorim da Costa Santos; Ana Carolina Simionato; Felipe Augusto Arakaki

Definição de metadados para recursos informacionais: apresentação da metodologia BEAM

asseguram a preservação e principalmente, otimizam o fluxo informacional melhorando o acesso aos dados e a localização dos recursos informacionais.

Para descrever essas funções, Gilliland-Swetland (1999) apresentou os metadados em cinco níveis: administrativos (gerenciamento e administração dos recursos informacionais), descritivos (descrição e identificação de informações sobre recursos), de conservação (conservação do recurso), técnicos (funcionamento do sistema e comportamento dos metadados) e de uso (nível e tipo de uso do recurso).

Os objetivos da catalogação se completam com o uso de metadados na tarefa de representação da forma e do conteúdo de um recurso informacional, pois o seu uso pressupõe uma padronização que atuará na facilidade da identificação para o acesso, a busca, a localização e a recuperação de recursos (HSIEH-YEE, 2006).

Contudo, é importante destacar que a catalogação é mais abrangente, não se restringindo apenas ao tratamento descritivo da informação (TDI) e a apresentação dos atributos de um recurso para a sua representação, sua função é de gestora na modelagem dos catálogos e de bancos de dados para os ambientes informacionais focados nas tarefas do usuário e os metadados são elementos integrantes dos serviços e produtos gerados no processo de catalogação (FUSCO, 2010; SIMIONATO, 2012).

Nesse sentido, os catálogos e bancos de dados necessitam de um planejamento que considere desde a camada mais interna, a camada intangível, até a camada de apresentação, a camada tangível, aquela a que o usuário tem acesso ao uso, considerando que a "[...] estrutura de representação de recursos informacionais encontra-se na camada intangível dos sistemas de informação e ao usuário o acesso se dá na camada aparente do sistema: na camada de apresentação." (SANTOS, 2010, p. 222).

$\mathrm{Na}$ implementação de metadados, deve ser realizada uma análise de domínio, ou seja, devem ser criadas estruturas que garantam a utilização de padrões de descrição e que favoreçam a interpretação de cada metadado. Sendo que esses metadados atuarão como possíveis pontos de acesso tanto ao recurso isoladamente, quanto aos seus relacionamentos com outros recursos informacionais.

Nessa perspectiva e na busca por diminuir as anomalias no sistema, o modelo conceitual Functional Requirements for Bibliographic Records (FRBR) é 
Plácida Leopoldina Ventura Amorim da Costa Santos; Ana Carolina Simionato; Felipe Augusto Arakaki

Definição de metadados para recursos informacionais: apresentação da metodologia BEAM

apresentado como uma orientação para a definição de requisitos funcionais na construção de registros bibliográficos, durante a etapa de abstração na modelagem de catálogos e bancos de dados bibliográficos.

Na modelagem dos catálogos será necessário definir a camada intrínseca, de persistência dos dados, e nela estarão estabelecidas as propriedades e níveis de descrição, as características de representação do domínio de conhecimento, as características do tipo de suporte e a padronização adotada, considerando o que se intenciona oferecer aos usuários.

A camada da persistência dos dados é aquela onde se garante 0 armazenamento não-volátil de dados e é de grande relevância para o tratamento descritivo da informação (TDI), pois ela estabelecerá a consistência e a integridade que se requer na descrição do recurso de modo que seu registro descritivo possa ser inserido em diferentes contextos. (SIMIONATO, 2012).

Ou seja, nessa camada os metadados têm como função a representação das entidades definidas na abstração para a modelagem conceitual do sistema e os atributos têm como objetivos atender os requisitos definidos na construção do catálogo, pois os modos de representação também interferem na capacidade de identificação do registro.

\section{METODOLOGIA PARA A DEFINIÇÃO DOS METADADOS}

A definição dos metadados para catalogação de recursos informacionais, visa à escolha dos elementos necessários para descrever os diferentes tipos de recursos.

Essa atividade tem como objetivo definir os elementos descritivos que farão parte do registro que representa cada item que compõe o acervo de uma determinada instituição e assim promover a gestão de acervos e de coleções pautada nos objetivos institucionais, no domínio do conhecimento e nas tarefas do usuário.

O processo é trabalhoso, mas traz grandes benefícios para a catalogação, evitando ambiguidade na descrição e equívocos na escolha do software para a gestão de bibliotecas. 
Plácida Leopoldina Ventura Amorim da Costa Santos; Ana Carolina Simionato; Felipe Augusto Arakaki

Definição de metadados para recursos informacionais: apresentação da metodologia BEAM

O Quadro 1 apresenta alguns métodos para a definição de metadados.

Quadro 1 - Métodos para definição dos metadados
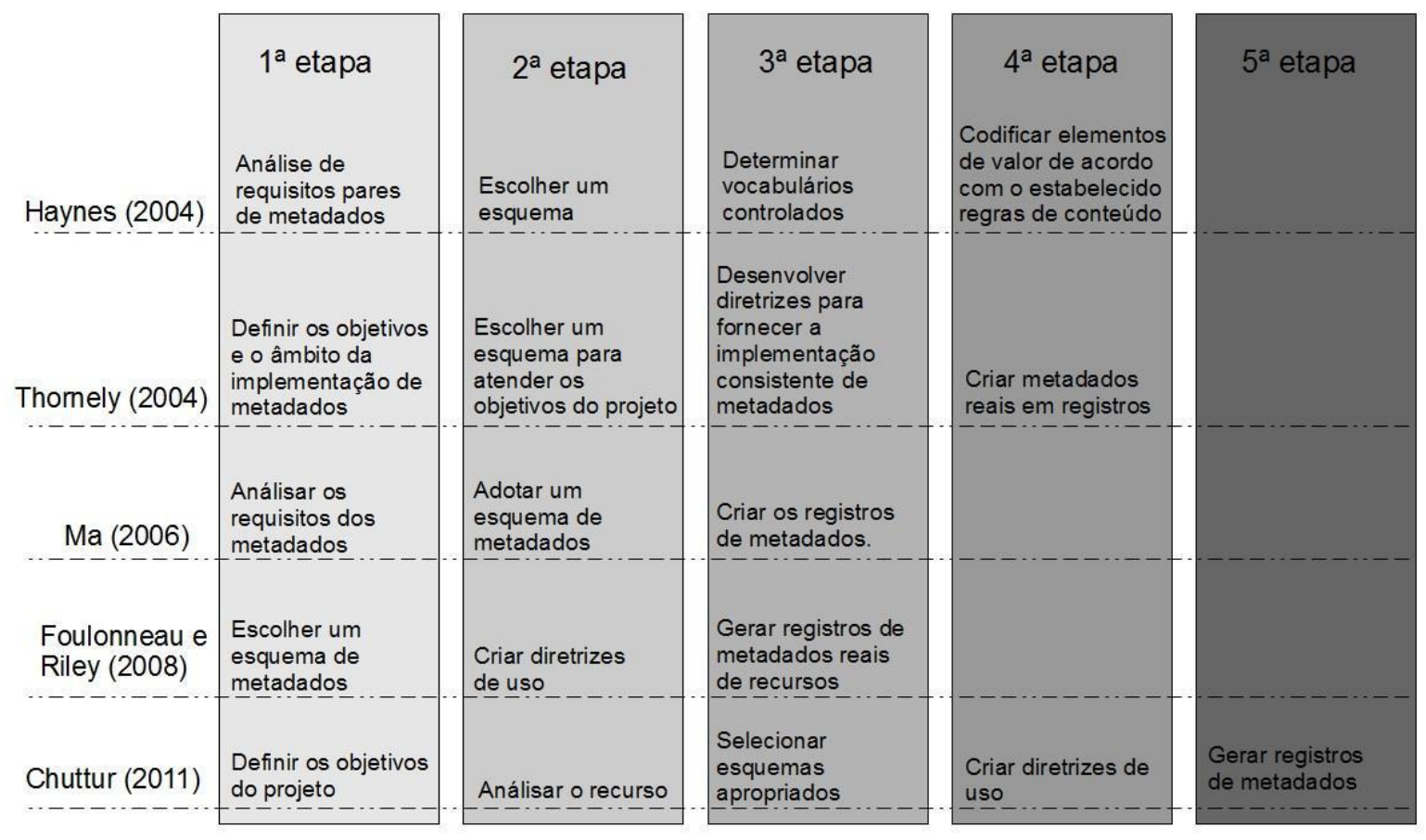

Fonte: Autores.

No estudo realizado e que gerou a síntese apresentada no Quadro 1, nota-se que não foi identificado um método bem definido e conciso que oriente a definição de metadados. Na busca por uma metodologia para a definição de metadados o modelo utilizado pelo grupo Data Observation Network for Earth (DataONE) parece oferecer alguns subsídios para a continuidade dos estudos. O DataONE é uma fundação cooperativa, com financiamento da National Science Foundation EUA (NSF) e que tem por objetivo servir de base para uma ciência inovadora que respeite as necessidades da sociedade para o acesso aberto aos dados.

A Figura 1, apresenta o ciclo de vida dos dados na perspectiva do pesquisador segundo o DataONE (2012) e oferece uma visualização para uma proposta de gerenciamento. 
Plácida Leopoldina Ventura Amorim da Costa Santos; Ana Carolina Simionato; Felipe Augusto Arakaki

Definição de metadados para recursos informacionais: apresentação da metodologia BEAM

Figura 1 - Ciclo de vida de dados na perspectiva de um pesquisador

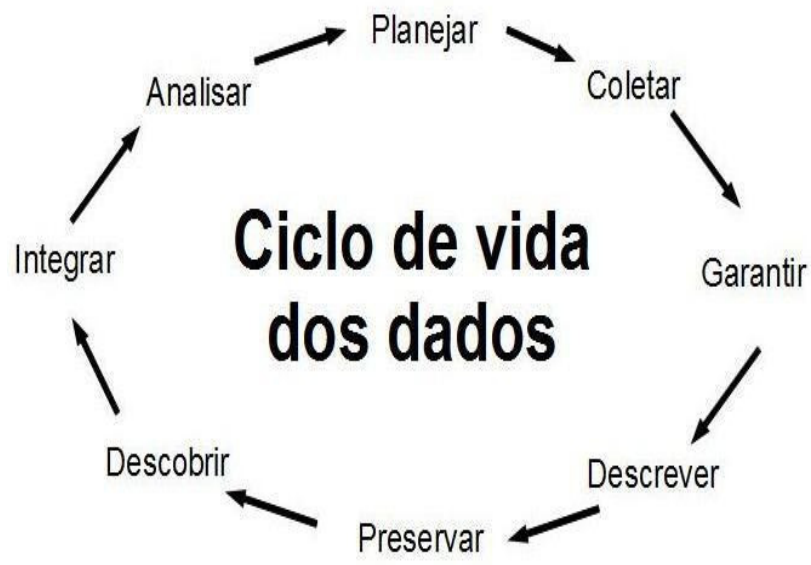

Fonte: DATAONE (2012, p. 3, tradução nossa).

O ciclo corresponde ao gerenciamento de dados e cada uma das partes que compõe esse ciclo é considerado como um processo que deverá ser respeitado durante a definição dos metadados. Os processos para o gerenciamento, segundo 0 DataONE (2012, p. 4) são:

- Planejar: processo inicial da definição, é nele que se criam estratégias e maneiras de como os dados serão coletados e gerenciados, pensando na forma em que serão acessados e em quanto tempo durará a definição. Considera-se, nesse processo, que uma série de padrões podem auxiliar na definição dos metadados: os elementos podem ser adicionados conforme às necessidades apresentadas pelo domínio, pelos usuários e pela própria instituição. Nessa fase, poderá ser utilizado um plano de ação, com um conjunto de questionamentos:

$\checkmark$ Quem?: A pessoa e/ou organização responsável pela coleta e tratamento dos dados;

$\checkmark \quad$ O que?: Os parâmetros medidos ou observados e quais são as unidades de suas medidas ou resultados;

$\checkmark$ Quando?: A descrição das características temporais de seus dados (por exemplo, o tempo de suporte, espaçamento e extensão);

$\checkmark$ Onde?: A descrição das características espaciais de seus dados e os detalhes para a sua implantação; 
Plácida Leopoldina Ventura Amorim da Costa Santos; Ana Carolina Simionato; Felipe Augusto Arakaki

Definição de metadados para recursos informacionais: apresentação da metodologia BEAM

$\checkmark$ Como?: Os métodos utilizados (por exemplo, instrumentos de análise);

$\checkmark$ Por quê?: O objetivo do estudo e da definição dos metadados;

$\checkmark$ Qualidade?: Os dados descritos interferirão na qualidade e/ou ajudarão outros a determinar se seus dados estão aptos para o fim ou não.

- Coletar: processo de coleta e de organização dos metadados realizado manualmente e mapeado de forma que sejam mais fáceis de observar os dados;

- Garantir: processo de controle de que todos os dados foram corretamente coletados;

- Descrever: processo de transcrição dos dados, exatamente como se apresentam e a utilização dos metadados apropriados para cada recurso;

- Preservar: processo em que os dados são submetidos a um arquivo adequado para sua preservação por um longo prazo (por exemplo, um banco de dados);

- Descobrir: processo de identificação dos dados potencialmente úteis, localizáveis e recuperáveis, juntamente com a informação relevante;

- Integrar: processo de integrar os dados com fontes diferentes para que sejam combinados, de modo que se mantenha a interoperabilidade;

- Analisar: processo final e, ao mesmo tempo, o recomeço, é a fase em que tudo o que foi planejado passa por uma revisão para verificar se as necessidades definidas foram atendidas.

Na revisão da literatura e na análise do ciclo de vida dos dados (DATAONE, 2012) destaca-se que o método de Chuttur (2011), apresentado na figura 1 , que orienta a definição de metadados em cinco fases: planejamento, análise do recurso, seleção de esquemas apropriados, criação de diretrizes de uso e de geração registros de metadados, não se faz suficiente.

Nesse sentido, apresenta-se a metodologia BEAM, desenvolvida tendo como ponto de partida o método de Chuttur (2011) e o ciclo de vida dos dados do DataONE (2012) apresentados anteriormente, e ainda o ciclo PDCA do método de gestão organizacional (CAMPOS, 1997, 2002, 2004) e a ferramenta de qualidade 
Plácida Leopoldina Ventura Amorim da Costa Santos; Ana Carolina Simionato; Felipe Augusto Arakaki

Definição de metadados para recursos informacionais: apresentação da metodologia BEAM

$5 \mathrm{~W} 1 \mathrm{H}$, que serão apresentaremos a seguir.

O ciclo PDCA é utilizado para o planejamento e a análise de processos de produção e de serviços. Ele se constitui em quatro fases, correspondentes às letras de sua sigla: to Plan, to Do, to Check e to Act, traduzidas para o português como: planejar, fazer, avaliar e agir, descritas como:

- Planejar: elaboração do plano de ação traçando estratégias e ações para resolver o problema ou atingir metas;

- Fazer: execução do plano de ação;

- Avaliar: verificação dos resultados esperados;

- Agir: correção e padronização dos processos avaliados.

Em conjunto do ciclo PDCA, propõe-se a utilização da ferramenta de qualidade $5 \mathrm{~W} 1 \mathrm{H}$ na fase do planejamento. $\mathrm{O}$ método consiste em organizar cada uma das atividades previstas no plano de ação, de modo a orientar os integrantes a responderem cada uma das questões, o que permitirá maior clareza das fases que serão desenvolvidas.

A ferramenta $5 \mathrm{~W} 1 \mathrm{H}$ consiste em apresentar respostas para algumas questões como apontado no modelo do DataONE, a partir das seguintes palavras-chave:

$\checkmark$ What - O que será feito e quais serão os itens de controle qualidade (etapas);

$\checkmark$ When - Quando e com que frequência cada uma das tarefas deverá ser executada (tempo);

$\checkmark$ Where - Onde cada uma das etapas será executada (local);

$\checkmark$ Who-Quem realizará as tarefas (responsabilidade);

$\checkmark$ Why-Por que a tarefa deve ser executada (justificativa);

$\checkmark$ How - Como deverá ser realizada cada tarefa e/ou etapa, como exercer o controle, indicar grau de prioridade para ação de cada um (método).

O estudo desses métodos orientou a definição dos metadados para a Biblioteca de Estudos e Aplicação de Metadados (BEAM) e a construção de uma metodologia que se inicia no planejamento do catálogo com a apresentação de um 
Plácida Leopoldina Ventura Amorim da Costa Santos; Ana Carolina Simionato; Felipe Augusto Arakaki

Definição de metadados para recursos informacionais: apresentação da metodologia BEAM

registro descritivo para os recursos disponíveis, contemplando o maior nível de descrição possível e que pode ser elemento importante na decisão de escolha de um software de gerenciamento para bibliotecas.

Nesse contexto, a metodologia desenvolvida compreende sete etapas:

1. Planejar - definição dos objetivos e análise de necessidades a partir das respostas para as perguntas do método $5 \mathrm{~W} 1 \mathrm{H}$. Elaborar o plano de ação traçando estratégias e ações para definir os metadados e atingir metas;

2. Executar - execução do plano de ações. Compreender claramente os objetivos institucionais e a complexidade dos recursos a serem representados, suas especificidades e identificação da existência de padrões de metadados para o mesmo propósito;

3. Seleção de esquemas para a definição de metadados - análise dos padrões de metadados atualizados e com abrangência de utilização nas instituições. Comparar os instrumentos de descrição previamente determinados. Identificar os elementos comuns nos esquemas, padrões e formatos de descrição. Criar elementos que garantam a especificidade do recurso;

4. Criar diretrizes de uso - elaboração das diretrizes de uso para a instituição, a partir do mapeamento dos dados realizado na etapa anterior, apontando a organização dos elementos e a correspondência dos metadados definidos, o esquema descrição de recursos e o formato de intercâmbio de dados;

5. Gerar registros de metadados - construção de registros de metadados;

6. Avaliar - análise de todos os pontos definidos na etapa do planejamento verificando os resultados esperados. Revisar todo o processo de definição dos metadados até alcançar o pressuposto inicial;

7. Ação para correções - correção resultante da análise e da padronização dos processos avaliados. 
Plácida Leopoldina Ventura Amorim da Costa Santos; Ana Carolina Simionato; Felipe Augusto Arakaki

Definição de metadados para recursos informacionais: apresentação da metodologia BEAM

Quadro 2 - Metodologia BEAM

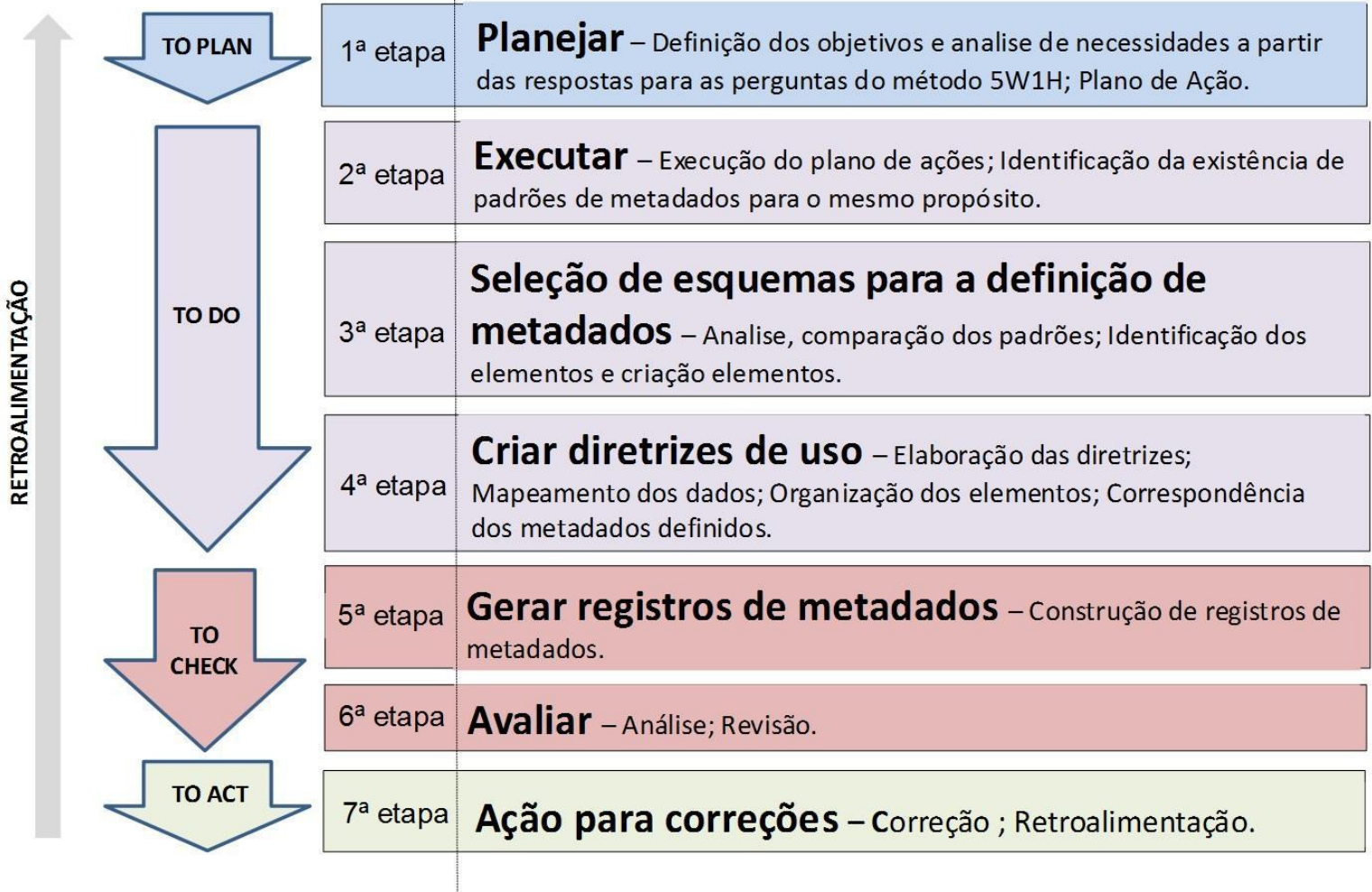

Fonte: Autores.

As etapas propostas se deram a partir do estudo dos diferentes tipos de recursos informacionais da BEAM: artigos científicos e livros, impressos ou em formato eletrônico.

Os esquemas de descrição utilizados para análise e definição dos metadados para a descrição dos recursos da BEAM foram: Anglo-American Cataloguing Rules, second edition (AACR2r), International Standard Bibliographic Description (ISBD) e Resource Description and Access (RDA) e o formato de intercâmbio de dados bibliográficos: Machine Readable Cataloging (MARC 21).

Vale ressaltar que não foi utilizado o padrão de metadados Dublin Core nesse trabalho, pois o propósito desse padrão é para uso geral e seus quinze elementos já são contemplados nos esquemas de descrição utilizados para a análise.

$\mathrm{Na}$ análise foi realizado um mapeamento das áreas do AACR2r, das zonas das ISBDs e das orientações RDA. Além dos campos e subcampos do formato MARC21 considerando também os metadados administrativos.

Essa etapa favoreceu a definição adequada dos atributos para a garantia de 
Plácida Leopoldina Ventura Amorim da Costa Santos; Ana Carolina Simionato; Felipe Augusto Arakaki

Definição de metadados para recursos informacionais: apresentação da metodologia BEAM

especificidade de diferentes tipos de itens informacionais, revelando, em algumas situações, a necessidade da inclusão de atributos não contemplados nos instrumentos convencionais da catalogação, mas que são necessários para a melhor descrição do recurso.

Contudo, ressalta-se que os instrumentos para orientações de descrição são utilizados para a definição dos metadados e também são diretamente relacionados ao tipo de recurso informacional a ser representado, ao domínio de conhecimento e às políticas institucionais, o que evidencia a importância do trabalho realizado nas três primeiras etapas da metodologia BEAM.

A elaboração de um manual de decisões é necessária, pois, nele são registradas todas as decisões do processo, os metadados definidos para a descrição dos diferentes tipos de recurso, o vocabulário controlado a ser utilizado, os registros de autoridade, além de diretrizes para o preenchimento dos valores dos metadados.

Por fim, a criação dos registros descritivos que representam o recurso em um software que suporte as necessidades informacionais da instituição. No caso da BEAM, a opção mais adequada foi o sistema Koha Integrated Library System, um software Open Source para o gerenciamento de bibliotecas, que em sua base de dados possibilita a modelagem dos metadados e a criação templates com os metadados definidos para cada tipo de recurso que se tenha a necessidade de representar.

\section{CONSIDERAÇÕES FINAIS}

Os ambientes informacionais digitais são caracterizados por um volume crescente na geração e uso de informações e no armazenamento de recursos, volume esse, que requer um tratamento especifico que otimize a utilização dos recursos, facilite o intercâmbio de dados, oportunize a interoperabilidade entre sistemas, garanta a consistência, a preservação dos dados armazenados e, de modo especial, ofereça eficiência na localização da informação desejada e na recuperação do recurso escolhido.

Nesse contexto, os metadados se configuram como peças fundamentais que influenciam tanto no acesso aos dados quanto na busca da informação, como 
Plácida Leopoldina Ventura Amorim da Costa Santos; Ana Carolina Simionato; Felipe Augusto Arakaki

Definição de metadados para recursos informacionais: apresentação da metodologia BEAM

também na localização de recursos e em sua recuperação, principalmente, aqueles disponíveis na Web.

A metodologia BEAM, desenvolvida na Biblioteca de Estudos e Aplicação de Metadados, no interior do Grupo de Pesquisa - Novas Tecnologias em Informação, da Unesp, para a definição de metadados para a catalogação de recursos informacionais apresenta-se como um importante instrumento para o planejamento de catálogos e bancos de dados, no processo de catalogação em uma biblioteca que se propõe à construção de registros com um alto nível de descrição para a representação qualificada de seus recursos e que tem seus objetivos voltados para as tarefas do usuário.

\section{REFERÊNCIAS}

ALVES, RACHEL CRISTINA VESÚ. Metadados como elementos do processo de catalogação. 2010. 132 f. Tese (Doutorado em Ciência da Informação)- Faculdade de Filosofia e Ciências, Universidade Estadual Paulista, Marília, 2010. Disponível em: <http://www.marilia.unesp.br/Home/Pos-

Graduacao/Cienciadalnformacao/Dissertacoes/alves_rcv_do_mar.pdf $>$. Acesso em: 17 fev. 2013.

BAPTISTA, Ana Alice; MACHADO, Altamiro Barbosa. Um gato preto num quarto escuro: falando sobre metadados. Revista de Biblioteconomia de Brasília, Brasília, v. 25, n. 1, p. 77-90, 2001. Disponível em:

<http://repositorium.sdum.uminho.pt/handle/1822/380>. Acesso em: 17 fev. 2013.

BORKO, Hilda. Information science: what is it? ABI/INFORM Globalpg, Washigton, v. 19, n. 1, p. 3-5, jan. 1968. Disponível em:

<http://pt.scribd.com/doc/39023567/Information-Science-What-is-It>. Acesso em: 17 fev. 2013.

CAMPOS, Vicente Falconi. Gerenciamento pelas diretrizes. Belo Horizonte: Fundação Christiano Ottoni, 1997.

Gerenciamento da rotina do trabalho do dia-a-dia. Belo Horizonte:

Desenvolvimento Gerencial, 2002.

Q.C: controle da qualidade total. 8. ed. Belo Horizonte: Fundação

Christiano, 2004.

CHUTTUR, Mohammad Yasser. Defining and creating metadata for digital resources.

Library Student Journal, Illinois, mar. 2011. Disponível em:

<http://librarystudentjournal.org/index.php/lsj/article/view/217/296>. Acesso em: 17 fev. 2013. 
Plácida Leopoldina Ventura Amorim da Costa Santos; Ana Carolina Simionato; Felipe Augusto Arakaki

Definição de metadados para recursos informacionais: apresentação da metodologia BEAM

COYLE, Karen; BAKER, Thomas. Guidelines for dublin core application profiles. 2009. Disponível em: <http://dublincore.org/documents/profile-guidelines/>.Acesso em: 16 fev. 2014.

DATAONE. Data Observation Network for Earth: Preserving Data and Enabling Innovation in the Biological and Environmental Sciences. Disponível em: $<$ http://www.dlib.org/dlib/january11/michener/01michener.html>. Acesso em: 17 fev. 2013.

FUSCO, Elvis. Modelos conceituais de dados como parte do processo da catalogação: perspectiva de uso dos FRBR no desenvolvimento de catálogos bibliográficos digitais. 2010. 249 f. Tese (Doutorado em Ciência da Informação)Faculdade de Filosofia e Ciências, Universidade Estadual Paulista, Marília, 2010. Disponível em:

<http://www.athena.biblioteca.unesp.br/exlibris/bd/bma/33004110043P4/2010/fusco_ e_dr_mar.pdf>. Acesso em: $17 \mathrm{fev} .2013$.

GILLILAND-SWETLAND, Ann J. La definición de los metadatos. In: BACA, Murtha et al. (Org.). Introducción a los metadatos: vías a la información digital. [S. I.]:

GETTY, 1999. p. 1-9.

GLUSHKO, Robert J. (Ed.). The discipline of organizing. Massachusetts: MIT Press, 2013.

HSIEH-YEE, Ingrid. Organizing audiovisual and electronic resources for access: a cataloging guide. Washington: Library of Congress, 2006.

NILSSON, Mikael; BAKER, Thomas; JOHNSTON, Pete. The singapore framework for dublin core application profiles. 2008. Disponível em:

<http://dublincore.org/documents/singapore-framework/>. Acesso em: 16 fev. 2014.

SANTOS, Plácida Leopoldina Ventura Amorim da Costa. Catalogação revisitada: sua história e contemporaneidade. 2010. 248 f. Tese (Livre-docência)-Faculdade de Filosofia e Ciências, Universidade Estadual Paulista, Marília, 2010.

SANTOS, Plácida Leopoldina Ventura Amorim da Costa; ALVES, Rachel Cristina Vesú (Org.). Biblioteca de estudos e aplicação de metadados. Disponível em: <https://sites.google.com/site/gpntibeam/>. Acesso em: 16 fev. 2014

SMIRAGLIA, Richard. Metadata: a cataloger's primer. Washington, DC: OUTLEDGE-USA, 2005.

SIMIONATO, Ana Carolina. Representação, acesso, uso e reuso da imagem digital. 2012. 141 f. Dissertação (Mestrado em Ciência da Informação)-Universidade Estadual Paulista "Júlio de Mesquita Filho", Faculdade de Filosofia e Ciências, Marília, 2012. Disponível em: <http://www.marilia.unesp.br/Home/PosGraduacao/Cienciadalnformacao/Dissertacoes/Simionato\%20A.C._mestrado_C.I__2 012.pdf>. Acesso em: 17 fev. 2013. 
Plácida Leopoldina Ventura Amorim da Costa Santos; Ana Carolina Simionato; Felipe Augusto Arakaki

Definição de metadados para recursos informacionais: apresentação da metodologia BEAM

ST. PIERRE, Margaret; LAPLANT, William P. Issues in crosswalking content metadata standards. Baltimore: NISO, 1998. Disponível em:

<http://www.niso.org/publications/white_papers/crosswalk>. Acesso em: 19 fev. 2013.

Title

Metadata definition for informational resources: presentation of methodology BEAM

\section{Abstract}

Introduction: In the digital environment, metadata influence both in data access and information retrieval and are used as search elements to facilitate locating resources on the Web.

Objective: In this perspective, the aim is to present the methodology BEAM, developed in Biblioteca de Estudos e Aplicação de Metadados, of the Research Group "Novas Tecnologias em Informação" in Universidade Estadual Paulista and used to define the metadata for describing information resources.

Methodology: The methodology used for the construction of the research is exploratory and bibliographic and was developed based on the theoretical method Chuttur (2011) and the life cycle of data from the DataOne (2012) and also the PDCA cycle and tool $5 \mathrm{~W} 1 \mathrm{H}$.

Results: The seven steps of the methodology are presented and also the necessary guidelines for their implementation.

Conclusions: We conclude pointing BEAM methodology that can be adopted by libraries in the construction of catalogs aimed at meeting the needs of users.

Keywords: Metadata. Automated cataloging. Modeling catalogs. Metadata definition. Information and technology.

\section{Titulo}

Definición de los metadatos para recursos de informacionales: presentación de la metodología BEAM

\section{Resumen}

Introducción: En el entorno digital, metadatos influyen el acceso a los datos y recuperación de la información y se utilizan como una búsqueda de elementos para facilitar la localización de los recursos en la Web.

Objectivo: En esta perspectiva, el objetivo es presentar la metodología BEAM, desarrollado en la Biblioteca de Estudos e Aplicação de Metadados, del Grupo de Investigación - Novas Tecnologias em Informação, de la Universidade Estadual Paulista y se utiliza para definir los metadatos para la descripción de recursos informacionales. 
Plácida Leopoldina Ventura Amorim da Costa Santos; Ana Carolina Simionato; Felipe Augusto Arakaki

Definição de metadados para recursos informacionais: apresentação da metodologia BEAM

Metodología: En este sentido, la metodología utilizada para la construcción de la investigación es exploratoria y bibliográfica, y fue desarrollado en base al método teórico Chuttur (2011) y el ciclo de vida de DataOne de datos (2012), así como el ciclo PDCA y la herramienta $5 \mathrm{~W} 1 \mathrm{H}$.

Resultados: Los siete pasos de la metodología se presentan, así como las orientaciones necesarias para su aplicación.

Concluciónes: Concluye señalando metodología BEAM que puede ser adoptado por las bibliotecas, en la construcción del catálogos destinados a satisfacer las necesidades de los usuarios.

Palabras clave: Metadatos. Catalogación automatizada. Modelado del catálogo. Definición de metadatos. Información y tecnología.

Recebido em: 19.06.2013

Aceito em: 25.02.2014 